\title{
Relaciones entre empatía y valores socialmente responsables en una muestra de estudiantes universitarios
}

\author{
Connections between empathy and socially responsible values, in a sample of \\ university students
}

\author{
Ana María Morales Rodríguez, Francisco Manuel Morales Rodríguez, Manuel Alejandro Narváez Peláez \\ Universidad de Málaga
}

\begin{abstract}
Resumen
Se analizan las relaciones entre la variable empatía y comportamientos socialmente responsables en una muestra 53 estudiantes universitarios de posgrado entre 24 y de 49 años de edad del Máster en Profesorado de Educación Secundaria de la Facultad de Ciencias de la Educación de la Universidad de Málaga a los que se les pasó un cuestionario para evaluar empatía y un cuestionario para evaluar comportamientos de responsabilidad social. Los resultados demuestran que existen correlaciones positivas entre las puntuaciones obtenidas en la variable empatía y las puntuaciones obtenidas en el cuestionario para evaluar comportamientos socialmente responsables en universitarios.

Palabras clave: empatía, universitarios, comportamiento socialmente responsable, competencia.
\end{abstract}

\begin{abstract}
The aim of this work is to analyse the relations between the empathy variable and the socially responsible behaviours. A sample of 53 postgraduate students, aged 24 to 49 , in "Educación Secundaria (junior high school) Teaching" at Educational Sciences Faculty, University of Málaga, have undertaken a survey consisting in two questionnaires: one to evaluate empathy, and the other to assess social responsibility behaviours. Results demonstrate that positive correlations exist between the empathy variable and the socially responsible behaviours marks received from the students.
\end{abstract}

Keywords: empathy, university students, socially responsible behaviour, competence.

El presente trabajo se enmarca en el Proyecto de Innovación Educativa (PIE13-031), en el que se plantea, entre otros aspectos, la necesidad de fomentar la adquisición y desarrollo de competencias transversales (por ejemplo, sociales y ciudadanas, de educación para la solidaridad y los derechos humanos, etc) desde una perspectiva holística dentro de una dimensión humanística/interpersonal y sociocultural.

Una de las variables más relevantes en dicha dimensión humanística/interpersonal es la empatía. En el contexto en el que se desarrolla este trabajo, en el ámbito universitario es necesario seguir estudiando las relaciones entre estas variables $y$ otras como el razonamiento moral, la conducta prosocial, eticidad, comportamientos y actitudes de responsabilidad social.

En investigaciones previas se pone de manifiesto que a mayor puntuación en la dimensión cognitiva de la variable empatía mayor es la predisposición a la autoatribución de comportamientos socialmente responsables (Martí-Noguera, Martí-Vilar y Almerich, 2014; Navarro et al., 2010).

En la misma línea, en otros trabajos (Arango-Tobon, Clavijo-Zapata, Puerta-Lopera y Sanchez-Duque, 2014; Fairchild, Van Goozen, Calder, Stollery y Goodyer, 2009) se señala cómo de algún modo los comportamientos socialmente responsables se fundamentan en el desarrollo empático de las personas.

Precisamente, el objetivo de este trabajo es analizar las relaciones entre la variable empatía y comportamientos socialmente responsables en una muestra estudiantes universitarios a los que se les aplicó un cuestionario para evaluar empatía y un cuestionario para evaluar comportamientos de responsabilidad social.

\section{Método}

\section{Participantes}

Los participantes han sido 53 estudiantes universitarios de posgrado entre 24 y de 49 años de edad del Máster en Profesorado de Educación Secundaria de la Facultad de Ciencias de la Educación de la Universidad de Málaga.

\section{Instrumentos o Materiales}

Los instrumentos que se han utilizado en el presente trabajo han sido los siguientes:

a) Cuestionario de empatía (Davis, 1980). La puntuación total se obtiene sumando respuestas tipo Liker de 5 opciones: $1=$ No me describe bien; $2=\mathrm{Me}$ describe un poco; $3=$ Me describe bien; 4= Me describe bastante bien; 5= Me describe muy bien. Consta de 28 ítems distribuidos en cuatro sub-escalas de 7 ítems cada una. Los ítems inversos se ponen con signo negativo (A mayor puntuación en esos ítems indican menor grado de empatía): $-3,-15,-7,-12,-4,-14,-18,-13,-19$.

b) Cuestionario de autoatribución de
comportamientos socialmente responsables en
universitarios (CACSR, Davidovich, Espina, Navarro y Salazar, 2005). Este instrumento incluye dos escalas: una de frecuencia y otra de intencionalidad. Está compuesto por un total de 40 ítems según escala tipo Likert de cinco escalones según se trate de la escala de frecuencia $(1=$ Nunca; 2= Casi nunca; 3=A veces; 4= Casi siempre y 5= Siempre) o la escala de intención (1= No aplica; $2=$ Beneficio personal; $3=$ No tengo clara la intención; $4=$ Beneficio para otros y $5=$ Beneficio mutuo). Ambas escalas están formadas por las siguientes diez categorías: 
Responsabilidad laboral/académica, actividades de voluntariado, ayuda social, actividades religiosas, convivencia social, responsabilidad cívica, autocuidado, desarrollo cultural, ecología y medio ambiente, y respeto por espacios compartidos.

Este instrumento es útil para evaluar la frecuencia de autoatribución de comportamiento socialmente responsable.

\section{Procedimientos}

Se ha insistido en la privacidad y en el carácter voluntario de la participación sin particularizar entre grupos o personas en el tratamiento de los datos. El CACSR y el cuestionario de empatía se aplicaron de forma colectiva dentro del horario académico establecido en el centro y fue administrado por un investigador.

\section{Resultados}

Los resultados demuestran que existen correlaciones positivas entre las puntuaciones obtenidas en la variable empatía y las puntuaciones obtenidas en el cuestionario para evaluar comportamientos socialmente responsables en universitarios.

A continuación se muestran en la Tabla 1 los resultados estadísticamente significativos más relevantes considerando la correlación existente entre cada ítem del cuestionario de empatía (a modo de estudio exploratorio inicial) y cada uno de los ítems que se corresponden o representa un comportamiento socialmente responsable determinado. Para organizar la información se presenta según el orden en que aparecen correlaciones estadísticamente significativas y con objeto de ser breves y concisos tan solo se muestran algunos ejemplos que se han seleccionado a modo de evidencias de las correlaciones existentes entre los ítems que representan un determinado comportamiento socialmente responsable con aquellos otros que representan acciones o aspectos correspondientes al constructo denominado empatía.

A continuación en dicha Tabla 1 , como ya se ha indicado, se muestran solo algunas de las correlaciones de dichos ítems que evidencian las relaciones entre los aspectos o constructos con los que se corresponden cada uno de dichos ítems, es decir, entre la variable empatía y comportamientos socialmente responsables.
Tabla 1.

Correlaciones de Pearson entre item que refleja acción o aspecto empática e items correspondiente a comportamiento socialmente responsable

\begin{tabular}{|c|c|}
\hline Ítems que correlacionan & Correlación \\
\hline Empatía 2 y Comp6 & $.32 *$ \\
\hline Empatía 3 y Comp5 & $.32 *$ \\
\hline Empatía 7 y Comp6 & $32 *$ \\
\hline Empatía 8 y Comp6 & $.53 * *$ \\
\hline Empatía 9 y Comp6 & $.38 * *$ \\
\hline Empatía 15 y Comp5 & $.51 * *$ \\
\hline Empatía 18 y Comp 4 & $.33 *$ \\
\hline Empatía 21 y Comp6 & $.37 * *$ \\
\hline Empatía 23 y Compl & $.30 *$ \\
\hline Empatía 24 y Comp5 & $.31 *$ \\
\hline Empatía 25 y Compl & $.39 * *$ \\
\hline Empatía 27 y Comp25 & $.29 *$ \\
\hline Empatía 28 y Compl & $.47 * *$ \\
\hline Empatía 28 y Comp6 & $.37 * *$ \\
\hline Empatía 1 y Compl1 & $.30^{*}$ \\
\hline Empatía 2 y Comp9 & $.34 *$ \\
\hline Empatía 6 y Comp12 & $-.31 *$ \\
\hline Empatía 8 y Comp9 & $.32 *$ \\
\hline Empatía 8 y Comp10 & $.32 *$ \\
\hline Empatía 9 y Comp7 & $.29 *$ \\
\hline Empatía 9 y Comp9 & $.40 * *$ \\
\hline Empatía 14 y Comp7 & $.33 *$ \\
\hline Empatía 15 y Comp12 & $-.28 *$ \\
\hline Empatía 17 y Comp7 & $-.29 *$ \\
\hline Empatía 18 y Comp10 & $-.35 * *$ \\
\hline Empatía 18 y Comp12 & $-.35 * *$ \\
\hline Empatía 19 y Comp10 & $.30^{*}$ \\
\hline Empatía 25 y Comp8 & $.29 *$ \\
\hline Empatía 25 y Comp11 & $.28^{*}$ \\
\hline Empatía 27 y Comp7 & $-.46 * *$ \\
\hline
\end{tabular}

$* * p<.01 ; * p<.05 ;$ EmpatíaX= ítems correspondientes al constructo empatía; CompX $=$ ítems correspondientes al constructo comportamiento socialmente responsable 


\section{Discusión y Conclusiones}

Los resultados obtenidos muestran la existencia de relaciones entre la variable empatía y la de comportamiento socialmente responsable.

Se concluye señalando la utilidad e importancia de estos datos en el Espacio Europeo de Educación Superior en el que es necesario seguir desarrollando competencias transversales (como las sociales y ciudadanas) planteadas en este proyecto así como en futuros programas de intervención para la mejora de la convivencia social, el trabajo a favor de otros y los derechos humanos donde es relevante fomentar variables como la empatía.

Además es necesario seguir profundizando en sus relaciones con otros constructos relacionados con la mayor predisposición y emisión de comportamientos socialmente responsables como se demuestra en este trabajo; considerando que el ámbito universitario constituye uno de los principales agentes dinamizadores para el fomento de una educación en valores para la cooperación, la solidaridad, la igualdad, la equidad y el fomento de los derechos humanos en las actuales sociedades que pretenden ser democráticas.

En este sentido, en el ámbito universitario cada vez son más los estudios interesados en el análisis de comportamiento socialmente responsables (Bustamante y Navarro, 2009; Martí-Noguera, Martí-Vilar y Almerich, 2014) dada su relevancia para las distintas reflexiones y propuestas didácticas que pueden encontrarse (por ejemplo, se pueden consultar trabajos como Amnistía Internacional, 1995; Naval y Herrero, 2006; Naya, 2005) para formar ciudadanos europeos y educar para la solidaridad y los Derechos Humanos en las actuales sociedades que pretenden ser democráticas. Otros autores como De la Herán (2000) señalan que en las actuales sociedades democráticas la educación en valores como la solidaridad cobra un sentido fundamental que comienza observando al estudiante, logrando la empatía a través de una cordialidad saludable y que intenta responder a la formación que posibilite el crecimiento personal. Para ello incide en la importancia de la transversalidad como estrategia para la formación en el ámbito universitario (De la Herrán, 2000).

\section{Referencias}

Amnistía Internacional (1995). Educación en Derechos Humanos. Propuestas didácticas. Madrid: Sociedad Española de Amnistía Internacional.

Arango-Tobon, O. E., Clavijo-Zapata, S. J., Puerta-Lopera, I. C. y Sanchez-Duque, J. W. (2014). Formación académica, valores, empatía y comportamientos socialmente responsables en estudiantes universitarios. Revista de la Educación Superior, 43(169), 89-105. Recuperado de:

http://www.redalyc.org/articulo.oa?id $=604307$ 53006

Bustamante, M. y Navarro, G. (2009). Autoatribución de comportamientos socialmente responsables en estudiantes de carreras del área de Ciencias Sociales. Revista Perspectiva, 18, 45-121.
Recuperado de:

http://dialnet.unirioja.es/descarga/articulo/2573 403.pdf

Davidovich, M. P., Espina, A., Navarro, G. y Salazar, L. (2005). Construcción y estudio piloto de un cuestionario para evaluar comportamiento socialmente responsables en estudiantes universitarios. Revista de Psicología de la Universidad de Chile, 14(1),125-139.

Davis, M. (1980). A multidimensional approach to individual differences in empathy. Catalog of Select Documents in Psychology, 10, 85-100. Recuperado de: http://www.uv.es/friasnav/ Davis 1980.pdf

De la Herrán, A. (Coord.) (2003). Guías didácticas para la formación de maestros. Huelva: Hergué Editorial.

Fairchild, G., Van Goozen, A. J., Calder, S. J., Stollery, S. \& Goodyer, I. (2009). Deficits in facial expression recognition in male adolescents with early-onset or adolescence-onset conduct disorder. The Journal of Child Psychology and Psychiatry, 50(5),627-636. doi:10.1111/j.1469-7610.2008.02020x.

Marti-Noguera, J. J., Martí-Vilar, M. y Almerich, G. (2014). Responsabilidad Social Universitaria: Influencia de valores y empatía en la autoatribución de comportamientos socialmente Responsables. Revista Latinoamericana de Psicología, 46(3), 160-168.

Naval, C. y Herrero, M. (Eds.). (2006). Educación y Ciudadanía en una sociedad democrática. Madrid: Ediciones Encuentro.

Navarro, G., Boero, P., Jiménez, G., Tapia, L., Hollander, R., Escobar, A., ... Espina, A. (2010). Universitarios y responsabilidad social. Calidad de la Educación, 33, 101-121.

Naya, L. M. (Coord.) (2005). La educación y los derechos humanos. Donostia: Erein. 